this effect and correlates better with degree of end-organ damage. Now, Verberk et al. have demonstrated that prescription on the basis of self-measured BP reduces the use of antihypertensive drugs without compromising BP control or exacerbating target organ damage.

In this prospective, randomized trial, 430 adults whose office-measured BP was at least $140 / 90 \mathrm{mmHg}$ were randomized to receive treatment on the basis of either self-measured BP (six times per day in a sitting position; average of 39 measurements in a 1-week period; $n=216)$ or office-measured BP $(n=214)$. Median durations of follow-up were 354 days and 351 days, respectively.

A significantly greater proportion of patients with self-measured BP than with officemeasured BP were able to permanently discontinue their medication because their BP dropped below $140 / 90 \mathrm{mmHg}(11 \%$ vs $2 \% ; P<0.0001)$. After 1 year, no significant differences in absolute office-measured BP or change in office-measured BP were found between the two groups; however, ambulatory values were higher in the self-measurement group. Both groups showed significant reductions from baseline in urinary microalbumin concentration and left ventricular mass $(P<0.001$ for both).

Original article Verberk WJ et al. (2007) Self-measurement of blood pressure at home reduces the need for antihypertensive drugs: a randomized, controlled trial. Hypertension 50: 1019-1025

\section{High-dose atorvastatin improves renal function in patients with coronary heart disease}

Analysis of randomized controlled trials that investigated the impact of statins on coronary heart disease risk has indicated a possible nephroprotective effect. Shepherd et al. performed a subanalysis of the Treating to New Targets study data to investigate the effect of atorvastatin therapy on renal function over a 5-year period.

A group of 10,001 patients with stable coronary heart disease and LDL cholesterol levels $<3.37 \mathrm{mmol} / \mathrm{l}(130 \mathrm{mg} / \mathrm{dl})$ were randomly assigned to double-blind daily therapy with either 10 or $80 \mathrm{mg} /$ day atorvastatin as the sole lipid-lowering medication. Glomerular filtration rate (GFR) was estimated at baseline (mean approximated $65 \mathrm{ml} / \mathrm{min} / 1.73 \mathrm{~m}^{2}$ in both groups) and at the end of follow-up.

After 5 years, mean GFR had increased by $3.5 \mathrm{ml} / \mathrm{min} / 1.73 \mathrm{~m}^{2}$ in the lower dose group, and by $5.2 \mathrm{ml} / \mathrm{min} / 1.73 \mathrm{~m}^{2}$ in the group receiving $80 \mathrm{mg}$ atorvastatin per day $(P<0.0001)$. More patients on the higher dose showed an improvement in GFR to $>60 \mathrm{ml} / \mathrm{min} / 1.73 \mathrm{~m}^{2}$ (i.e. improvement in those with chronic kidney disease; $46 \%$ vs $38 \%, P=0.001$ ); $80 \mathrm{mg} /$ day atorvastatin was also associated with a decreased likelihood of GFR dropping to below $60 \mathrm{ml} / \mathrm{min} / 1.73 \mathrm{~m}^{2}(P<0.0001)$ in participants with normal renal function at baseline.

As well as being associated with fewer major cardiovascular events, superior lipid control and a favorable safety profile, dose escalation of atorvastatin is nephroprotective in people with coronary heart disease.

Original article Shepherd J et al. (2007) Effect of intensive lipid lowering with atorvastatin on renal function in patients with coronary heart disease: the Treating to New Targets

(TNT) study. Clin J Am Soc Nephrol 2: 1131-1139

\section{Increasing prevalence of CKD in the US}

A comparison of data from two surveys of large, nationally representative samples of adults in the US general population - the National Health and Nutrition Examination Surveys (NHANES) 1988-1994 and 1999-2004-shows that the prevalence of chronic kidney disease (CKD) has increased between the two periods.

In this study, CKD was staged according to the NKF-K/DOQI guidelines (stage 1: persistent albuminuria and estimated GFR [eGFR] $>90 \mathrm{ml} / \mathrm{min} / 1.73 \mathrm{~m}^{2}$; stage 2: persistent albuminuria and eGFR $60-89 \mathrm{ml} / \mathrm{min} / 1.73 \mathrm{~m}^{2}$; stage 3 : eGFR $30-59 \mathrm{ml} / \mathrm{min} / 1.73 \mathrm{~m}^{2}$; stage 4 : eGFR $15-29 \mathrm{ml} / \mathrm{min} / 1.73 \mathrm{~m}^{2}$; stage 5 patients were excluded). Persistent albuminuria was defined as albumin:creatinine ratio $\geq 30 \mathrm{mg} / \mathrm{g}$ for $\sim 2$ weeks, and GFR was estimated using the abbreviated MDRD Study equation, recalibrated to standardized creatinine measurements.

The prevalences of albuminuria and decreased eGFR increased substantially with time. The prevalence rate of CKD increased from $10.0 \%$ (95\% Cl 9.1-10.9\%) in 1988-1994 to $13.1 \%(95 \% \mathrm{Cl} 12.0-14.1 \%)$ in $1999-2004$; rises occurred in each CKD stage but were greatest for stages $2-4$. Mean serum creatinine 